\title{
Primary Placement of a Titanium Sleeve in Hydroxyapatite Orbital Implants
}

\begin{abstract}
Background: To study a new surgical option of primary placement of a titanium sleeve into hydroxyapatite implants during enucleation or evisceration.

Methods: A standard enucleation or cornea preserved evisceration was performed, followed by preplacement of a titanium sleeve into the hydroxyapatite implant by a hand drill sleeve driver. Care must be taken to ensure that the titanium sleeve is positioned centrally when the implant is put inside the orbital socket or eviscerated shell. The Tenon capsule and conjunctiva were meticulously closed with minimal tension. Complications such as sleeve exposure, coralline exposure, and infection of the titanium sleeve were closely observed.
\end{abstract}

Results: In all, 30 patients were treated in the above fashion with 10 enucleation and 20 evisceration procedures. The follow-up period ranged from 9 to 24 months. Three of the sleeves were found to have exposed spontaneously at 5 and 7 weeks following original surgery. They had no further complication except one sleeve loosening. The remaining 27 sleeves that did not spontaneously expose pursued secondary exposure of the titanium sleeve and peg insertion by conjunctival cutdown procedure 3 months after original surgery. Two sleeves were found to be oblique positioned after the conjunctival cutdown procedure. Fortunately, all the 30 patients were successfully fit with a peg-coupled prosthesis with good motility.

Conclusion: Primary placement of a titanium sleeve into hydroxyapatite implants has several advantages, including high patient acceptance, technical simplicity, and officebased conjunctival cutdown pegging procedure. By avoiding the expense of postoperative imaging study and additional prosthetic modification, a more rapid and efficient rehabilitation is possible.

Eye (2005) 19, 400-405. doi:10.1038/sj.eye.6701509

Published online 6 August 2004

Keywords: hydroxyapatite implant; titanium sleeve; enucleation; evisceration

\section{Introduction}

Hydroxyapatite orbital implants ${ }^{1-3}$ are commonly used during enucleation, evisceration, and secondary orbital implant surgery. ${ }^{4-5}$ This implant is made of material similar in nature to the mineral component of human bone (calcium phosphate), is biocompatible, nontoxic, and nonallergic. ${ }^{1,2,5}$ Its extensive pore system permits fibrovascular ingrowth, which helps the implant to resist migration and infection. By attaching the extraocular muscles and coupling the prosthesis to the orbital implants using sleeve and peg, a wide range of prosthetic movements can be obtained, ${ }^{1,2,5}$ which allows for a more lifelike quality in the prosthetic eye.

Despite its successes, there are some challenges to the hydroxyapatite coupling system. A second surgical procedure is required to anchor the sleeve and peg, and it is sometimes difficult to place and align the peg precisely on the surface of the hydroxyapatite implant. An additional procedure of placing the motility peg in any setting may result in increased complications with the implant (eg, implant exposure around the peg) and with the peg itself (eg, peg extrusion and pyogenic granuloma formation) ${ }^{6,7}$ Finally, the cost of adjunctive radiological imaging (bone, computed tomographic, or magnetic resonance imaging scan) to confirm implant vascularization (if needed in some special cases), a second surgical procedure to place the peg system, and postoperative prosthesis modification are also high. It may be the topic of

\author{
All authors do not have any \\ financial interest on the \\ materials discussed in this \\ manuscript. \\ This paper was presented at \\ Ophthalmic Plastic and \\ Reconstructive Surgery \\ meeting 2002 as a poster. \\ manuscript. \\ Received: 5 October 2003 \\ Accepted in revised form: 9 \\ February 2004 \\ Published online: 6 August
}


interest to develop some technical modifications to simplify the procedure of placing the motility peg and achieve early-coupled prosthetic fitting.

Rubin et al (2000) developed a technique to place a motility coupling post (MCP) into porous polyethylene orbital implants at the time of enucleation. , $^{8}$ There were no infections, wound dehiscences, malpositions or extrusion of the posts in their series. Only minor complications of pyogenic granuloma (two cases) and conjunctival overgrowth (one case) were noted. He concluded that MCP placement at the time of enucleation in selected patients is an effective and efficient surgical option. ${ }^{9}$ In order to facilitate early coupling and simplify the pegging procedure, we place a titanium sleeve into a hydroxyapatite orbital implant at the time of enucleation or evisceration and planned a peg placement 3 months later.

\section{Patients and methods}

\section{Patient selection}

All patients who underwent primary placement of the titanium sleeve were highly motivated for early-coupled prosthetic fitting. The exclusion criteria included underlying vasculopathy (diabetes, vasculitis, or history of chemotherapy or radiotherapy), endophthalmitis, and patients younger than 15 years. Intraoperative requirements included adequate conjunctiva to close the wound without tension.

\section{Surgical technique}

In the case of enucleation, immediately following a standard enucleation, the posterior Tenon was widely opened with blunt dissection. The implant was wrapped with vicryl mesh (Ethicon, Somerville, NJ). Before placement into the orbital socket, an 18-gauge needle was used to prepare a pilot hole into the vicryl meshwrapped hydroxyapatite implant, (Integrated Orbital Implants, San Diego, CA), and a hand drill sleeve driver (Integrated Orbital Implants, San Diego, CA) was used to preplace a titanium sleeve (Integrated Orbital Implants, San Diego, CA) into the implant. The head of the titanium sleeve was positioned to protrude $1-3 \mathrm{~mm}$ above the surface of the hydroxyapatite implant.

(Figure 1) After preplacement of a titanium sleeve into the hydroxyapatite implant, the implant was slid into an orbital socket, while the anterior conjunctiva or Tenon capsule was retracted. A $1.5 \times 1.5 \mathrm{~cm}^{2}$ donor sclera was put on the top of sleeve with a buttonhole for the sleeve. (Figures 1 and 2) The four rectus muscles were sutured $3-5 \mathrm{~mm}$ from the anterior surface of the sleeve onto the donor sclera and vicryl mesh. The Tenon capsule and conjunctiva were meticulously closed with minimal

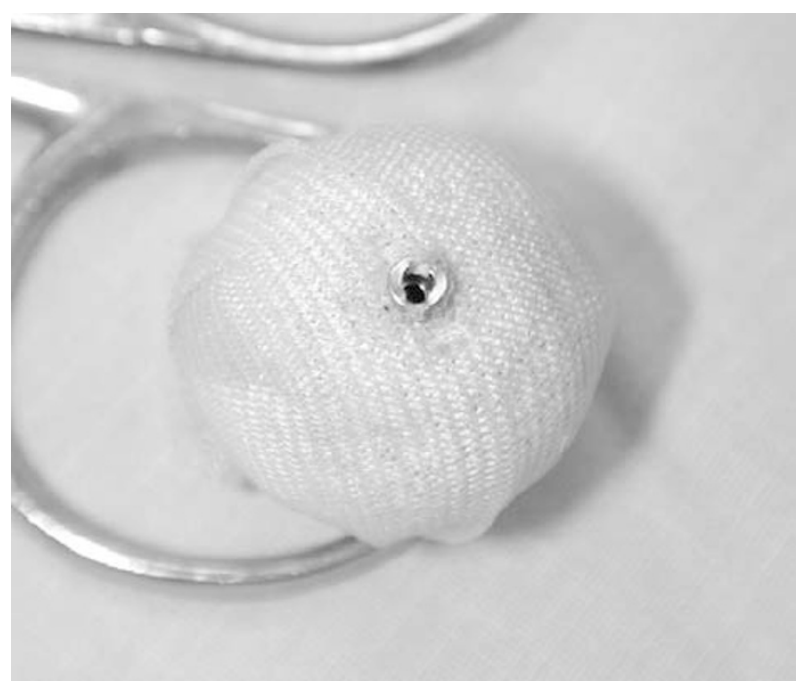

Figure 1 Vicryl mesh-wrapped hydroxyapatite orbital implant with sleeve.

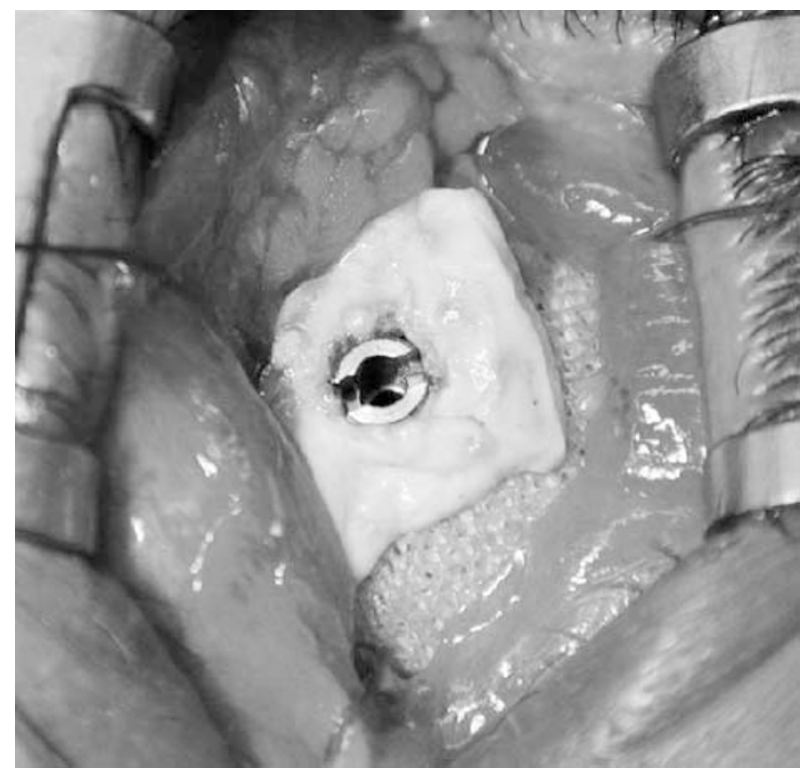

Figure 2 Hydroxyapatite orbital implant with sleeve and donor sclera on top was put into an ophthalmic socket.

tension using an interrupted buried 6-0 polyglactin suture. A polymethyl methacrylate conformer was placed within conjunctival fornices, antibiotic ointment was applied, and the eyelids were typically closed with a central suture tarsorrhaphy.

In the case of evisceration, a cornea preserved standard evisceration was performed, followed by preplacement of a titanium sleeve into unwrapped hydroxyapatite implant (as described above). The implant was placed in the eviscerated shell if the space was large enough. If the space of the eviscerated shell is not enough, a further 
circular opening of the scleral shell posterior to the equator is required to let the posterior part of the implant come out into the orbital space. Care must be taken to ensure that the titanium sleeve is positioned centrally when the implant is put inside the eviscerated scleral shell. Closure of the sclera-corneal wound was performed with 5-0 polyester sutures. The $2-3 \mathrm{~mm}$ buttonhole of the central cornea was created to let the titanium sleeve protrude out of the surface of the cornea. The Tenon capsule and conjunctiva were closed over the cornea in the same fashion as that in the enucleation procedure. A conformer was put in the conjunctival fornix and terramycin ointment was applied.

\section{Postoperative management}

Routine examinations were performed at 1 week, 1 month and 2 months after surgery. Patients were monitored postoperatively for the evidence of spontaneous exposure of the sleeve, infection, and early exposure of the hydroxyapatite implant. Secondary exposure of the sleeve and the peg insertion was scheduled 3 months after initial surgeries.

\section{Secondary exposure of the sleeve}

Topical anaesthesia was instilled into the conjunctival cul-del-sac, followed by a cotton pledget soaked in $2 \%$ lidocaine. The sleeve was palpated and conjunctival cautery or cutdown procedure was applied to expose the titanium sleeve (Figure 3) The peg was inserted into the central opening of the sleeve. No suturing of the conjunctiva was required. Topical antibiotics were given, and peg-coupled prosthetic fitting was scheduled 2 weeks after this procedure.

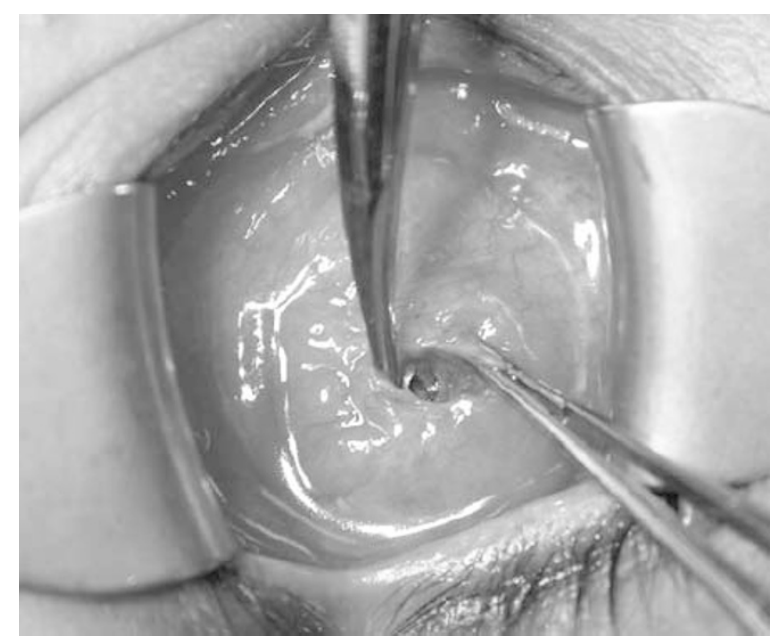

Figure 3 The conjunctival cutdown procedure was performed to expose the titanium sleeve 3 months after original surgery.

\section{Follow-up schedules}

All the patients were followed up 1 week after original surgery and every month for the following 6 months. After 6 months, patients were asked to come back every 3-6 months.

IRB/Ethics Committee approval was obtained in this study.

\section{Results}

In all, 30 patients were treated in the above fashion with 10 enucleation and 20 evisceration procedures from June, 2000 to January, 2002. The follow-up period ranged from 9 to 24 months (mean, 16 months). All the operations and follow-ups were performed by one doctor (Dr SL Liao) . Three of the sleeves (one enucleation, two eviscerations) were found to have exposed spontaneously at 5 and 7 weeks following the original surgery( Figure $4 \mathrm{a}, \mathrm{b})$. They had no further complications except one sleeve loosening. All three patients had a titanium peg insertion without a conjunctival cutdown procedure and were fit with a peg-coupled prosthesis 3 months after surgery.
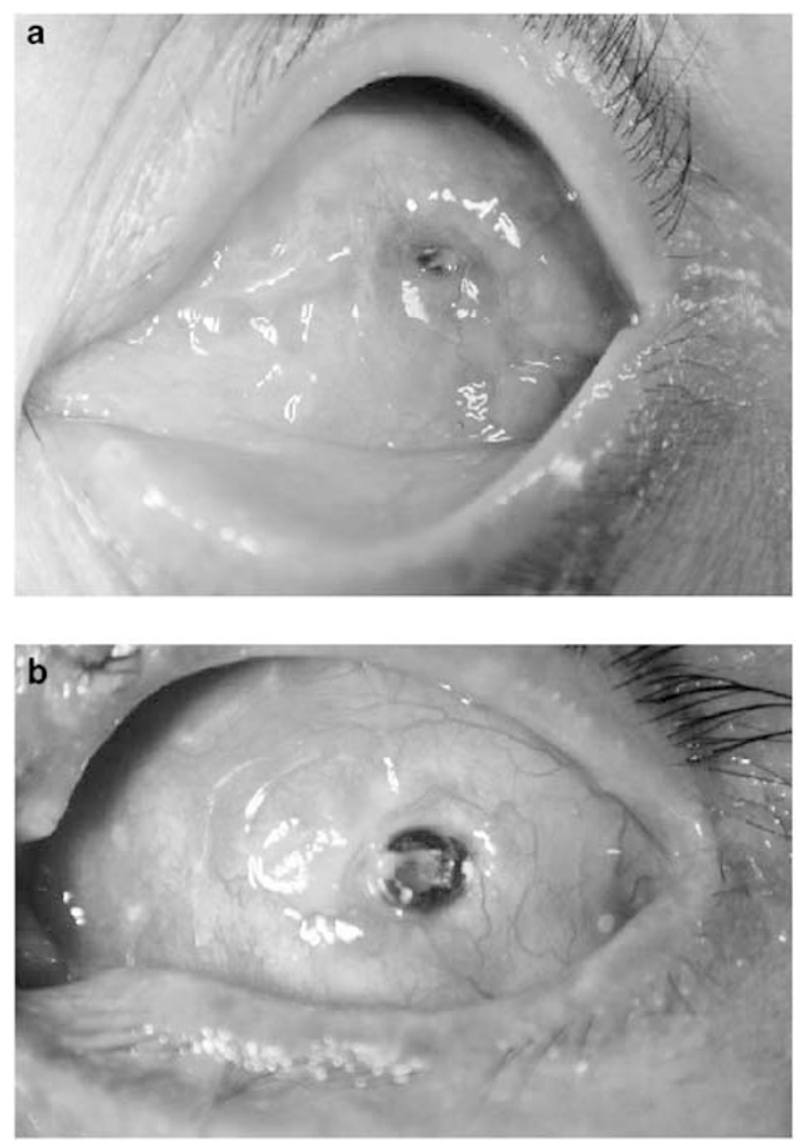

Figure 4 a, b: Two of the sleeves were found to have exposed spontaneously 5 and 7 weeks following original surgery. 


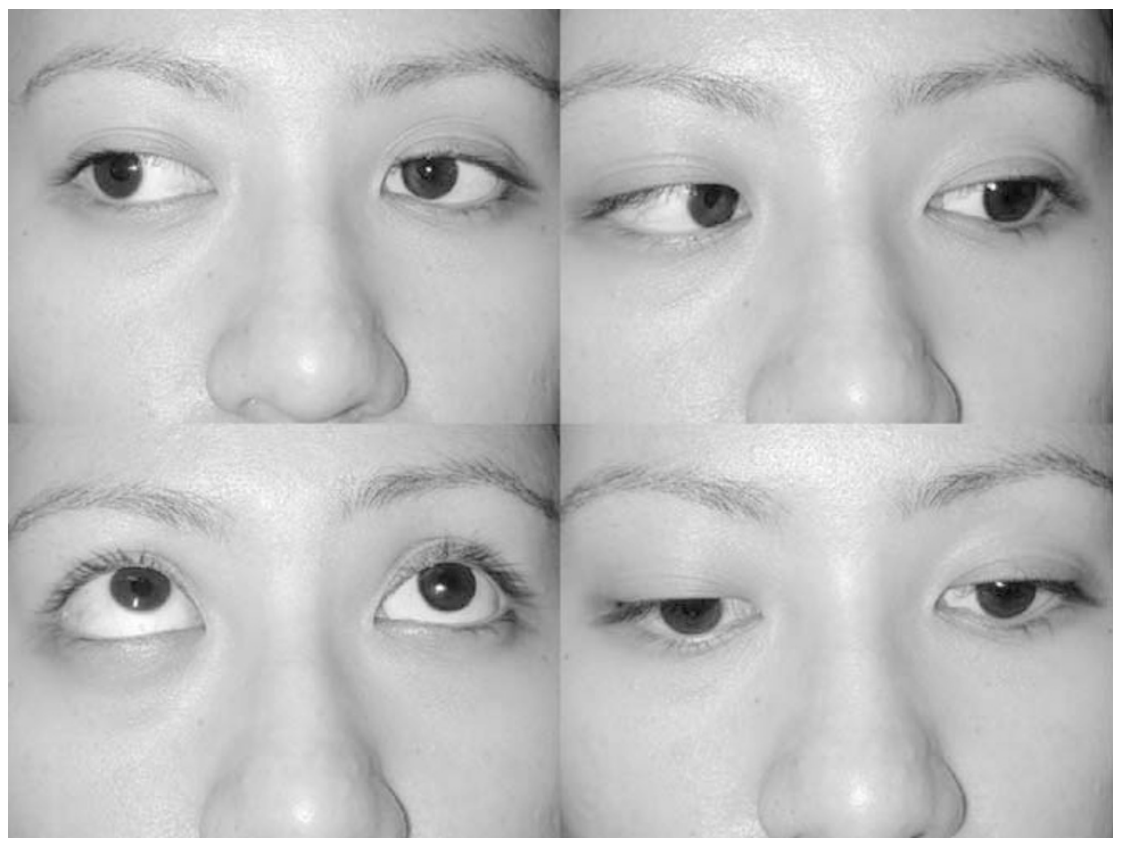

Figure 5 28-year-old female with primary sleeve placement during evisceration and early prosthetic fitting 3 months after surgery; the movement of prosthesis is very satisfactory.

The remaining 27 sleeves did not spontaneously expose. All these 27 patients pursued secondary exposure of the titanium sleeve and peg insertion by conjunctival cutdown procedure 3 months after original surgery. All the patients were successfully fit with a peg-coupled prosthesis with good synchronized motility (Figure 5).

\section{Major complications}

There were no wound dehiscences, infection, or extrusions of the titanium sleeve. Two sleeves were found to be oblique positioned after conjunctival cutdown procedure. Fortunately, these two patients could be fit with a peg-coupled prosthesis with acceptable synchronized motility. No patient required explantation of the implant or repositioning of the sleeve.

\section{Minor complications}

Among the 3 cases with spontaneous exposure of the titanium sleeve, sleeve loosening with hydroxyapatite spicules visible around the sleeve was noted in one case. No further hydroxyapatite exposure was found after 16 months of follow-up and this patient complained of increased discharge from the socket.

\section{Discussion}

Porous hydroxyapatite has been successfully used as an orbital implant in enucleation, evisceration, and as secondary implant since $1985 .^{1,2,4,10-12}$ The benefit of using this implant is its extensive porous system permitting fibrovascular ingrowths, which decreases the risk of implant extrusion and infection. ${ }^{2,13,14}$ In addition, with drilling and peg insertion, this implant can be directly coupled to the prosthesis, allowing a wide range of prosthetic movement, especially fine darting eye movements commonly seen during conversational speech. ${ }^{5}$ These movements impart a more lifelike quality to the prosthetic eye. ${ }^{5}$ However, peg placement is usually delayed (usually at least 6 months) until the implant shows a high degree of fibrovascularization ingrowths, as established by some objective imaging study, such as bone scan or magnetic resonance imaging scan. ${ }^{15,16}$ Because the cosmetic and psychological rehabilitation of the anophthalmic patients may depend on lifelike fine movements of the prosthetic eye, searching for new techniques to facilitate earlier coupled prosthetic fitting is beneficial to patients.

Rubin et al (2000) developed a technique to place an MCP into porous polyethylene orbital implants at the time of enucleation. ${ }^{9}$ Nine out of 32 MCPs (28\%) spontaneously exposed within the first 4 months, and there were no cases of infection, explantation, or gross MCP malposition in their series. In this study, we tried to place a titanium sleeve at the time of enucleation or evisceration. Three out of 30 sleeves (10\%) spontaneously exposed within the first 2 months after original surgery. Rubin et $a l^{9}$ found that pegs positioned less than $3 \mathrm{~mm}$ above the implant did not spontaneously expose, while 
pegs protruding $4 \mathrm{~mm}$ above the implant were much more likely to expose. ${ }^{9}$ In our case, we placed the sleeve $1-3 \mathrm{~mm}$ above the implant; that is why there was less sleeve exposure (10\% as compared to $28 \%$ ) in our study. The early exposure was not associated with major complications such as implant exposure, peg extrusion, or infection in this series. Only one sleeve loosening with hydroxyapatite spicules visible around the sleeve was found with increased discharge from the socket. Fortunately, no further hydroxyapatite exposure around the sleeve was found after 16 months of follow-up. Even though there is no further hydroxyapatite exposure for this case, we think that it would be better for the cases with early sleeve exposure to wait for a longer time to receive peg insertion, or more frequent outpatient visits should be followed if early peg insertion is really needed. Furthermore, in cases of primary placement of the sleeve or MCP, migration or rotation of the implants between its intraoperative and final postoperative position may result in decentration or misdirection of the sleeve or MCP. In Rubin's report, there was no malposition of MCP. ${ }^{9}$ In our study, two sleeves were found to be oblique positioned after conjunctival cutdown procedure. Fortunately, these two patients could be fit with a pegcoupled prosthesis with acceptable synchronized movements, and no more repositioning of the sleeve is needed.

The advantages of motility peg placement include increasing prosthetic motility (including small-angle conversational movement) and provide support of the prosthesis, which decreases the mechanical burden of the prosthesis on the lower eyelid. Several potential complications can occur after pegging the hydroxyapatite implant, including pyogenic granuloma, clicking sound, profuse discharge, conjunctival overgrowing peg, peg drilled on an angle and implant infection etc. ${ }^{7,17,18}$ In our study, all patients including 27 patients pursuing secondary exposure of the sleeve by conjunctival cutdown procedure and 3 patients with sleeve spontaneous exposure received peg-coupled prosthesis fitting. None of our patients experienced pyogenic granuloma, clicking sound, conjunctival overgrowing peg, and further implant infection. We also found that discharge from sockets with primary placement of the sleeve was much less than those with secondary placement of the sleeve and peg system in our previous report. ${ }^{18}$ It is possible that early vascular ingrowths around the primarily inserted sleeve may decrease discharge formation. Nevertheless, there were two sleeves with oblique position and one loosening sleeve noted in our series. Fortunately, these patients could be fit with a peg-coupled prosthesis without any further complications.
In conclusion, primary placement of the titanium sleeve is offered as a potential surgical option. Our study demonstrates the ease and predictability of this procedure with minimal complications. In selected patients, this procedure offers several advantages, including high patient acceptance, technical simplicity, and office-based conjunctival cutdown pegging procedure. By avoiding the expense of postoperative imaging study (to evaluate vascularization), secondary pegging surgery, and additional prosthetic modification and fitting, a more rapid and efficient rehabilitation is possible. Due to a rather short follow up time, additional long-term observations following primary placement of the titanium sleeve during enucleations or eviscerations are required.

\section{References}

1 Perry AC. Integrated orbital implants. Adv Ophthalmic Plast Reconstr Surg 1990; 8: 75-81.

2 Dutton JJ. Coralline hydroxyapatite as an ocular implant. Ophthalmology 1991; 98: 370-377.

3 Jordan DR, Gilberg S, Mawn L, Brownstein S, Grahovac SZ. The synthetic hydroxyapatite implant: a report on 65 patients. Ophthal Plast Reconstr Surg 1998; 14: 250-255.

4 Hornblass A, Biesman BS, Eviatar JA. Current techniques of enucleation: a survey of 5,439 intraorbital implants and a review of the literature. Ophthal Plast Reconstr Surg 1995; 11: 77-88.

5 Jordan DR. Anophthalmic orbital implants. Ophthalmol Clin North Am 2000; 13: 587-608.

6 Remulla HD, Rubin PAD, Shore JW, Sutula FC, Townsend DJ, Woog JJ et al. Complications of porous spherical orbital implants. Ophthalmology 1995; 102: 586-593.

7 Jordan DR, Chan S, Mawn L, Gilberg S, Dean T, Brownstein $\mathrm{S}$ et al. Complications associated with pegging hydroxyapatite orbital implants. Ophthalmology 1999; 106: 505-512.

8 Hsu WC, Green JP, Spilker MH, Rubin PA. Primary placement of a titanium motility post in a porous orbital implant: animal model with quantitative assessment of fibrovascular ingrowths and vascular density. Ophthal Plast Reconstr Surg. 2000; 16: 370-379.

9 Rubin PAD, Fay AM, Remulla HD. Primary placement of a motility coupling post in porous polyethylene orbital implants. Arch Ophthalmol 2000; 118: 826-832.

10 Shields CL, Shields JA, De Potter P, Singh AD. Problems with the hydroxyapatite orbital implant: experience with 250 consecutive cases. Br J Ophthalmol 1994; 78: 702-706.

11 De Potter P, Shields CL, Shields JA, Singh AD. Use of the hydroxyapatite ocular implant in the pediatric population Arch Ophthalmol 1994; 112: 208-212.

12 Massy GG, Holds JB. Coralline hydroxyapatite spheres secondary orbital implants in anophthalmos. Ophthalmology 1995; 102: 161-166.

13 Shields CL, Shields JA, De Potter P. Hydroxyapatite orbital implant after enucleation: experience with initial 100 consecutive cases. Arch Ophthalmol 1992; 110: 333-338.

14 Shields CL, Shields JA, Eagle Jr RC, De Potter P. Histopathologic evidence of fibrovascular ingrowth four 
weeks after placement of the hydroxyapatite orbital implant. Am J Ophthalmol 1991; 111: 363-366.

15 Baumgarten D, Wojno T, Taylor Jr A. Evaluation of biomatrix hydroxyapatite ocular implants with technetium99m-MDP. J Nucl Med 1993; 34: 467-468.

16 De Potter P, Shields C, Shields J, Flanders AE, Rao VM. Role of magnetic resonance imaging in the evaluation of the hydroxyapatite orbital implant. Ophthalmology 1992; 99: 824-830.
17 Edelstein C, Shields CL, De Potter P, Shields JA. Complications of motility peg placement for the hydroxyapatite orbital implant. Ophthalmology 1997; 104: 1616-1621.

18 Chun-Ju Lin, Shu-Lang Liao, Jieh-Ren Jou, Kao SC, Hou PK, Chen MS. Complications of motility peg placement for porous hydroxyapatite orbital implants. $\mathrm{Br} J$ Ophthalmol. 2002; 86: 394-396. 